\title{
Acute generalized exanthematous pustulosis with severe organ dysfunction
}

\author{
Marc-André Leclair MD, Bruno Maynard MD, Catherine St-Pierre MD
}

Previously published at www.cmaj.ca

$\mathrm{A}$ 53-year-old man was transferred to our intensive care unit for a generalized exanthem with systemic inflammatory response syndrome. His medical history included a possible disseminated infection of herpes zoster 15 years before the current admission. He had no history of psoriasis, cutaneous drug reaction, immune suppression or heart disease. Before the current incident, he had not been using any prescribed or over-the-counter medications.

Seven days before admission, the patient was bitten on his finger by a spider. He reported pain at the site, and there was a necrotic crust. His primary care physician prescribed cefprozil. A few hours after the first dose, a rash developed. The rash was described as a generalized exanthem with nonfollicular superficial pustules and fever. Based on a working diagnosis of recurrent herpes zoster, he was given famciclovir.

Two days before admission to our intensive care unit, the patient was admitted to another centre with high fever, fatigue and persistent skin lesions. Because of fever, leukocytosis and pustules, an infectious cause was suspected. Antibiotic coverage was broadened to include piperacillin-tazobactam and moxifloxacin. The results of tests for infectious and immunosupressive diseases were negative. A computerized tomography (CT) scan of the patient's chest showed alveolar opacities with a ground-glass appearance in both upper lungs. A CT scan of the patient's abdomen showed small nonspecific opac-

Table 1: Patient's laboratory tests results

\begin{tabular}{lcc}
\hline Test & Reference range & Result \\
\hline Leukocyte count, $\times 10^{9} / \mathrm{L}$ & $4.5-11$ & 26.4 (with left shift) \\
\hline Eosinophil count, $\times 10^{9} / \mathrm{L}$ & $0-0.2$ & 1.3 \\
\hline Lactate, $\mathrm{mmol} / \mathrm{L}$ & $0.7-2.1$ & 2.36 \\
\hline Albumin, $\mathrm{g} / \mathrm{L}$ & $35-50$ & 26 \\
\hline Sodium, $\mathrm{mmol} / \mathrm{L}$ & $135-145$ & 130 \\
\hline Calcium, $\mathrm{mmol} / \mathrm{L}$ & $2.10-2.54$ & 1.81 \\
\hline AST, U/L & $8-39$ & 78 \\
\hline ALT, U/L & $9-52$ & 121 \\
\hline Troponin T, $\mu \mathrm{g} / \mathrm{L}$ & $0-0.03$ & 1.56 \\
\hline
\end{tabular}

Note: $\mathrm{AST}=$ aspartate aminotransferase, $\mathrm{AL}=$ alanine aminotransferase

\section{Key points}

- Acute generalized exanthematous pustulosis is a rare severe cutaneous drug reaction that has a rapid onset after exposure.

- Sterile nonfollicular pustules found mainly in the major skin folds are characteristic.

- The most common causative agents are aminopenicillins, macrolides and quinolones.

- In almost all patients, recovery is spontaneous and complete after withdrawal of the causative drug.

ities in his liver. The patient was then transferred to our centre.

When the patient arrived at our centre, he had a fever $\left(39.9^{\circ} \mathrm{C}\right)$ and was tachycardic $(130$ beats $/ \mathrm{min})$ and hypotensive (90/80 $\mathrm{mm} \mathrm{Hg})$. His oxygen saturation was $90 \%$ on room air. There was no jugular venous distension. The results of a cardiac examination were unremarkable, except for tachycardia. More than $80 \%$ of his skin, including his face and scalp, was affected by a generalized erythema with multiple small nonfollicular pustules. An examination of his mucous membranes showed redness of his tongue. He did not have the Nicolsky sign. (The Nikolsky sign is detected by applying pressure to the skin, which causes intraepidermal cleavage that allows the superficial skin to slip free from the deeper layer. It is mainly seen with bullous diseases and toxic epidermal necrolysis.)

Important laboratory results are shown in Table 1. An electrocardiogram showed only sinus tachycardia. We suspected a cutaneous drug reaction. The main diagnoses considered at that time were Stevens-Johnson syndrome or toxic epidermal necrolysis and acute generalized exanthematous pustulosis associated with severe systemic inflammatory response syndrome. The results of the CT scan of the patient's chest were suggestive of an acute lung injury. An infectious cause was not likely because all of the cultures were negative. Antibiotics were discontinued, and fluid resuscitation was initiated. A few hours after admission, our patient developed pulmonary edema with acute respiratory failure. Noninvasive positive pressure ventilation and diuretic ther-

From the Intensive Care Unit, Department of Medicine (Leclair, St-Pierre), Centre Hospitalier Universitaire de Sherbrooke; and the Dermatology Division (Maynard), Centre Hospitalier Universitaire de Sherbrooke, Sherbrooke, Que.

Cite as CMAJ 2009. DOI:10.1503/cmaj.090137 
apy were successfully started. An echocardiography performed the next day showed reduced left ventricular ejection fraction of $30 \%$ (normal > 60\%) with diffuse hypokinesis.

A skin biopsy showed spongiform intraepidermal pustules and acanthosis, dermal edema with mixed inflammatory cells
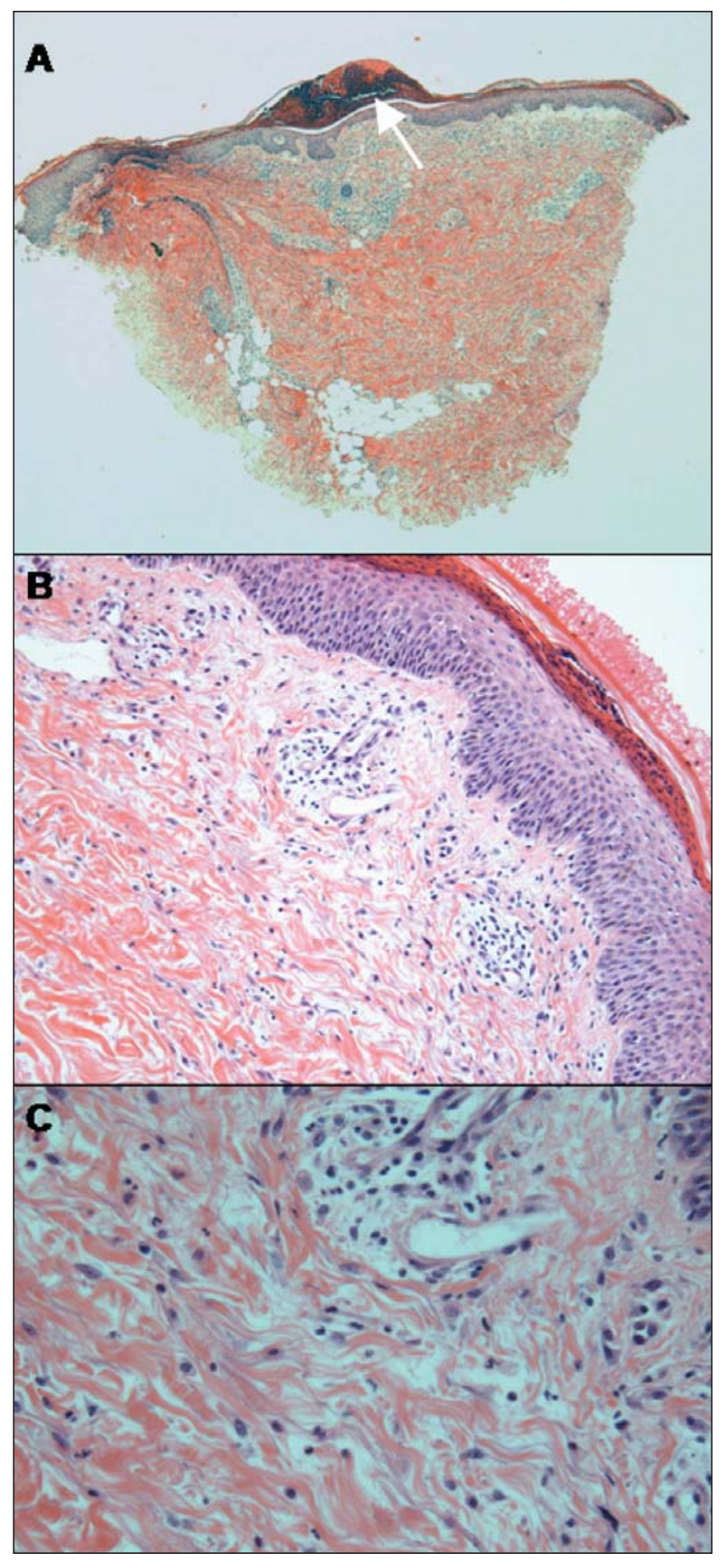

Figure 1: Histological sections of skin biopsy showing (A) a superficial epidermal pustulation (arrow), (B) and (C) edematous papillary dermis with mixed inflammatory cell infiltrate in the dermis (hematoxylin-eosin stain, original magnification $\times 4$ $[A], \times 20[B], \times 40[C])$. and neutrophilic perivascular infiltrates (Figure 1). We determined the likelihood of acute generalized exanthematous pustulosis by use of the EuroSCAR study group's scoring system, ${ }^{1}$ which can be used to identify cases of acute generalized exanthematous pustulosis based on morphology, course and histology of the skin reaction (Table 2). Our patient had a score of 11, which indicates a definite diagnosis of acute generalized exanthematous pustulosis.

Within 72 hours after discontinuing antibiotics, our patient's left ventricular ejection fraction normalized. The cutaneous lesions resolved within a few days, followed by widespread desquamation. Because of his clinical recovery, we decided not to give glucocorticoid therapy.

\section{Discussion}

Severe drug-induced skin reactions are a major diagnostic challenge. Stevens-Johnson syndrome and toxic epidermal necrolysis are well-described entities. (Stevens-Johnson syndrome is considered a mild form of toxic epidermal necrolysis.) However, acute generalized exanthematous pustulosis is an uncommon drug-related eruption that also warrants recognition. In the literature, it has different names, such as toxic pustuloderma, pustular drug rash and pustular psoriasiform eruption with leukocytosis. The EuroSCAR study group recently reported an annual incidence of $1-5$ cases per 1 million people. ${ }^{1}$ The

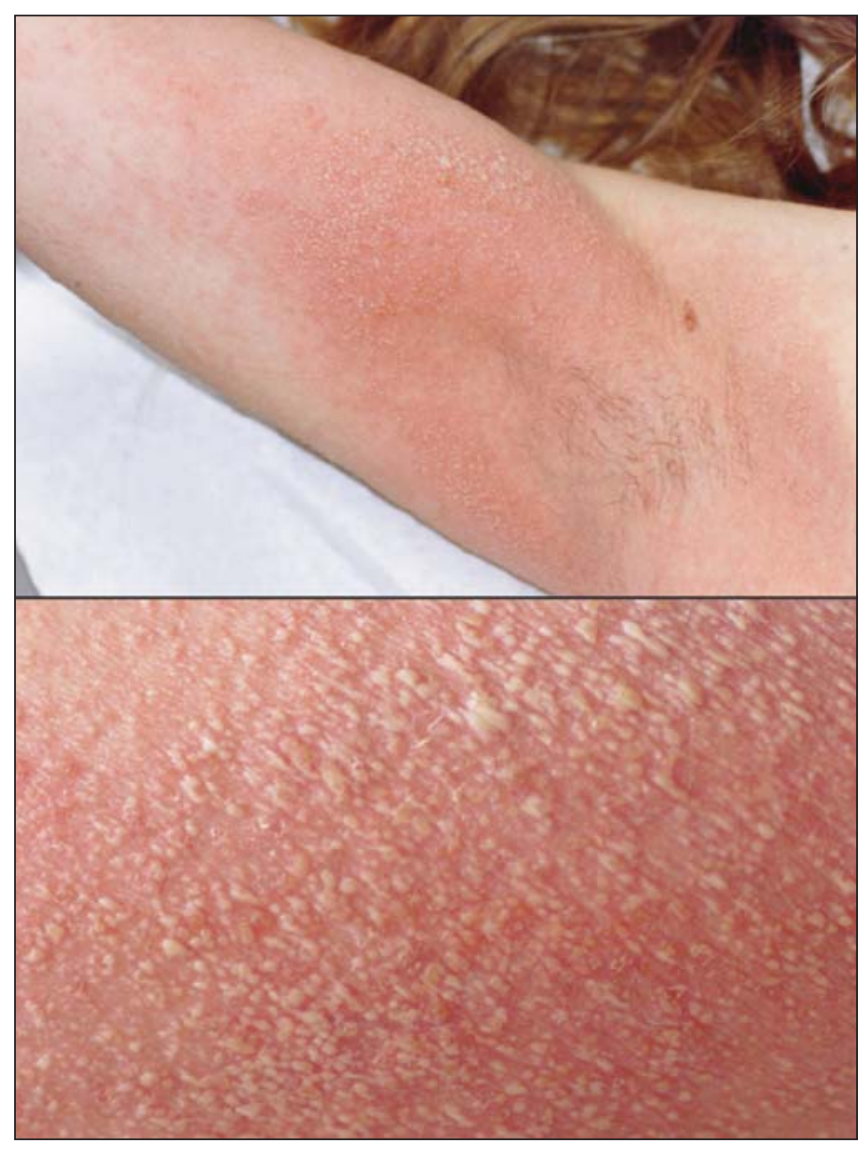

Figure 2: Typical pustular rash in acute generalized exanthematous pustulosis. Reproduced with permission from J Cutan Pathol. ${ }^{1}$ 
average age of onset is 56 years, and women are affected more often than men. ${ }^{2}$

\section{Clinical findings}

Acute generalized exanthematous pustulosis is characterized by superficial sterile pustules with spontaneous healing. The cutaneous eruption usually begins with edematous erythema in the intertriginous areas or in the face that disseminates acutely with burning and itching. Dozens of pinhead-sized nonfollicular pustules develop against the background of this erythema (Figure 2). The rash is often accentuated in the major skin folds. Mucous membrane involvement occurs in fewer than $20 \%$ of cases and is usually mild and limited to 1 region (usually the mouth). Cutaneous lesions resolve within a few days and are followed by a characteristic pin-point desquamation.

Skin biopsies typically show spongiform subcorneal or intraepidermal pustules, edema of the papillary dermis and perivascular infiltrates with neutrophils and exocytosis of some eosinophils. ${ }^{3}$ Vasculitis and necrosis of keratinocytes may be present but psoriasiform changes are absent.

Cutaneous manifestations are often accompanied by systemic symptoms such as high-grade fever, increased neutrophil count (in $80 \%$ of cases) and mild eosinophilia (in $30 \%$ of cases). ${ }^{4}$ Lymphadenopathy has also been reported. ${ }^{5}$ Dysfunction of the internal organs is not expected, although mild acute renal failure occurs in about one-third of patients. Mild hepatic dysfunction and acute renal failure that requires hemodialysis have also been reported. ${ }^{5}$ Dysfunction of multiple organ systems is rare, and only a few cases in the literature report hemodynamic disturbances. ${ }^{6-8}$ We believe that the abnormalities observed on the initial chest scans for our patient were related to cardiogenic pulmonary edema with systemic inflammatory response syndrome-associated increased vascular permeability. The severity and prolonged duration of the inflammatory response in our patient probably led to cardiac dysfunction.

\section{Causes}

More than $90 \%$ of cases of acute generalized exanthematous pustulosis are believed to be caused by drugs, most frequently by aminopenicillins, macrolides and quinolones. ${ }^{2}$ The syndrome has also
Table 2: The patient's score for acute generalized exanthematous pustulosis determined by use of the validation scoring system of the EuroSCAR study group

\begin{tabular}{|c|c|c|}
\hline Criteria & Description & Score* \\
\hline \multicolumn{3}{|l|}{ Morphology } \\
\hline Pustules & Typical, nonfollicular and sterile & +2 \\
\hline Erythema, $\%$ of body surface area & Typical, diffuse $(80 \%)$ & +2 \\
\hline Distribution or pattern & Compatible, trunk and limbs & +1 \\
\hline Postpustular desquamation & Yes & +1 \\
\hline Additional finding & No & \\
\hline Total score (0 to 7$)$ & & +6 \\
\hline \multicolumn{3}{|l|}{ Course } \\
\hline Erosive mucosal involvement & No & 0 \\
\hline Acute onset ( $\leq 10$ days) & Yes (few hours) & 0 \\
\hline $\begin{array}{l}\text { Resolution of pustules and } \\
\text { erythema } \leq 15 \text { days }\end{array}$ & Yes (10 days) & 0 \\
\hline Fever $\left(\geq 38^{\circ} \mathrm{C}\right)$ & Yes $\left(39.9^{\circ} \mathrm{C}\right)$ & +1 \\
\hline $\begin{array}{l}\text { Blood neutrophil count } \\
\left(\geq 7000 \text { cells } / \mathrm{mm}^{3}\right)\end{array}$ & Yes $\left(24000\right.$ cells $\left./ \mathrm{mm}^{3}\right)$ & +1 \\
\hline Total score $(-8$ to +2$)$ & & +2 \\
\hline \multicolumn{3}{|l|}{ Histology } \\
\hline Skin histology & $\begin{array}{l}\text { Spongiform intraepidermal pustules } \\
\text { with dermal edema; acanthosis; } \\
\text { perivascular infiltrate of neutrophils }\end{array}$ & +3 \\
\hline Total score $(-10$ to +3$)$ & & +3 \\
\hline Overall score $(-18$ to +12$)$ & & +11 \\
\hline
\end{tabular}

Note: EuroSCAR = European project dedicated to severe cutaneous adverse reactions.

*An overall score of -18 to 0 indicates no acute generalized exanthematous pustulosis; 1 to 4 indicates possible acute generalized exanthematous pustulosis; 5 to 7 indicates probable acute generalized exanthematous pustulosis; and 8 to 12 indicates definite acute generalized exanthematous pustulosis.

Table 3: Clinical features of Stevens-Johnsons syndrome or toxic epidermal necrolysis and acute generalized exanthematous pustulosis

\begin{tabular}{lcc}
\hline Characteristic & $\begin{array}{c}\text { Stevens-Johnsons syndrome } \\
\text { or toxic epidermal } \\
\text { necrolysis* }\end{array}$ & $\begin{array}{c}\text { Acute generalized } \\
\text { exanthematous } \\
\text { pustulosis* }\end{array}$ \\
\hline Onset of reaction & $1-3$ weeks & $<48$ hours \\
\hline Duration of eruption & $1-3$ weeks & $<1$ week \\
\hline Fever & +++ & +++ \\
\hline Facial edema & - & ++ \\
\hline Pustules & - & +++ \\
\hline Blisters & +++ & - \\
\hline Atypical target lesions & + & - \\
\hline Mucous membranes & +++ & - \\
\hline involvement & - & ++ \\
\hline Lymphadenopathy & ++ & +++ \\
\hline Hepatitis & - & + \\
\hline Leukocytosis & - & - \\
\hline Eosinophilia & & + \\
\hline
\end{tabular}

*One plus sign indicates that a characteristic occurs frequently, 2 plus signs indicate that a characteristic occurs very frequently, and 3 plus signs indicate that a characteristic always occurs. A minus sign indicates that the characteristic is absent. 


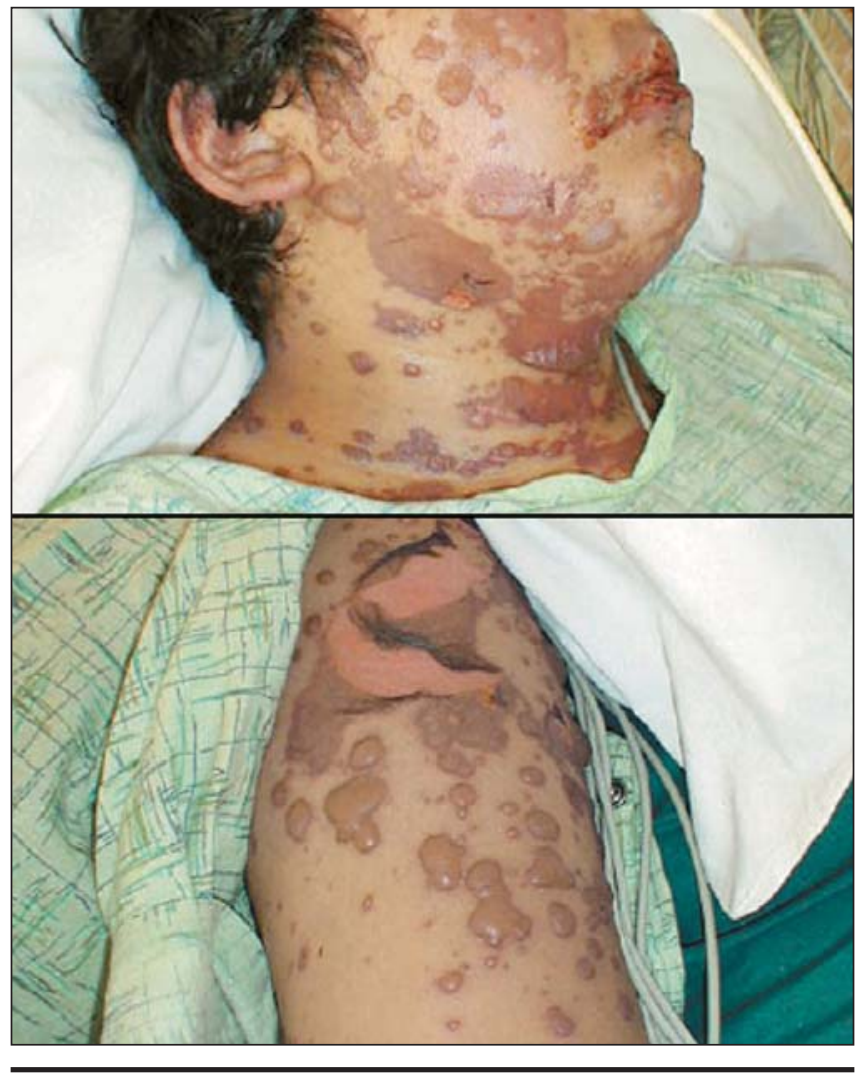

Figure 3: A patient with Stevens-Johnson syndrome and the Nikolsky sign. This rash began as with a maculopapular distribution on the patient's neck and chest and rapidly progressed to target lesions and bullae that involved all surfaces of the skin, sparing only the scalp. Reproduced with permission from the Massachusetts Medical Society. ${ }^{1}$

been described in relation to spider bites. ${ }^{9}$ Because our patient was exposed to cefprozil and was bitten by a spider, we cannot know with certainty what caused his reaction. Based on the temporal relation of the disease to drug exposure, we believe that cefprozil was the most likely cause.

The pathophysiological mechanisms of acute generalized exanthematous pustulosis are unclear, but an immunologic recall phenomenon has been suggested. Patch testing may help identify the responsible drug; however, a positive reaction was found in only 7 of 14 patients with acute generalized exanthematous pustulosis in a study of severe cutaneous drug reactions..$^{10}$

A cornerstone of therapy for drug-induced skin reactions is discontinuation of the causative drug. The overall prognosis for acute generalized exanthematous pustulosis is good with a self-limiting course. ${ }^{4}$ Glucocorticoids are not usually necessary because of the self-limiting course of the reaction.

\section{Differential diagnosis}

Physicians must differentiate between Stevens-Johnson syndrome or toxic epidermal necrolysis and acute generalized exanthematous pustulosis. Patients with Stevens-Johnson syndrome or toxic epidermal necrolysis should be treated like patients with major burns. A broad multidisciplinary team is often required to care for the extensive cutaneous, mucosal and ophthalmologic involvement. Sequelae, primarily dermatologic and ophthalmologic, can occur. Mortality, mainly because of sepsis, among patients with Stevens-Johnson syndrome or toxic epidermal necrolysis is as high as $35 \%-50 \%$, while the mortality is $5 \%$ among those with acute generalized exanthematous pustulosis. The clinical features that can be used to differentiate between these conditions are shown in Table 3 .

The clinical course of an acute generalized exanthematous pustulosis skin reaction is characteristic. ${ }^{2}$ Lesions usually occur within a few hours after exposure and resolve quickly over a few days. In contrast, there is usually a longer delay of 1 to 3 weeks after exposure in Stevens-Johnson syndrome and toxic epidermal necrolysis.

The confluence of the pustules in acute generalized exanthematous pustulosis may result in a positive Nikolsky sign and lead to an erroneous diagnosis of Stevens-Johnson syndrome or toxic epidermal necrolysis. Figure 2 and Figure 3 show characteristic rashes of acute generalized exanthematous pustulosis and Stevens-Johnson syndrome.

Acute generalized exanthematous pustulosis has a different spectrum of causative drugs then Stevens-Johnson syndrome and toxic epidermal necrolysis. Although antibiotics are important causes of both types of reactions, Stevens-Johnson syndrome and toxic epidermal necrolysis are associated more frequently with sulfonamides, and acute generalized exanthematous pustulosis is more frequently associated with aminopenicillins. Antiepileptic drugs and allopurinol do not play an important role in the pathogenesis of acute generalized exanthematous pustulosis, whereas they have been connected with Stevens-Johnson syndrome and toxic epidermal necrolysis. ${ }^{2}$

This article has been peer reviewed.

Competing interests: None declared.

\section{REFERENCES}

1. Sidoroff A, Halevy S, Bavinck JN, et al. Acute generalized exanthematous pustulosis - a clinical reaction pattern. J Cutan Pathol 2001;28:113-9.

2. Sidoroff A, Dunant A, Viboud C, et al. Risk factors for acute generalized exanthematous pustulosis (AGEP)-results of a multinational case-control study (EuroSCAR). Br J Dermatol 2007;157:989-96.

3. Burrows NP, Russell Jones RR. Pustular drug eruptions: a histopathological spectrum. Histopathology 1993;22:569-73.

4. Roujeau JC, Bioulac P, Bourseau C, et al. Acute generalized exanthematous pustulosis. Analysis of 63 cases. Arch Dermatol 1991;127:1333-8.

5. Eeckhout I, Noens L, Ongenae K, et al. Acute generalized exanthematic pustulosis a case with a lymphoma-like presentation. Dermatology 1997:194:408-10.

6. De Coninck AL, Van Strubarq AS, Pipeleers-Marichal MA, et al. Acute generalized exanthematous pustulosis induced by paracetamol: a case with severe hemodynamic disturbances. Dermatology 1996;193:338-41.

7. Lesterhuis WJ, Tjioe M, Stumpenhausen GA, et al. Acute generalized exanthematous pustulosis mimicking septic shock. Am J Med 2004;116:574-5.

8. Byerly FL, Nelson KC, Granko RP, et al. Valdecoxib-associated acute generalized exanthematous pustulosis. Burns 2005;31:383-7.

9. Davidovici BB, Pavel D, Cagnano E, et al. EuroSCAR; RegiSCAR study group. Acute generalized exanthematous pustulosis following a spider bite: report of 3 cases. J Am Acad Dermatol 2006;55:525-9.

10. Wolkenstein P, Chosidow O, Fléchet ML, et al. Patch testing in severe cutaneous adverse drug reactions, including Stevens-Johnson syndrome and toxic epidermal necrolysis. Contact Dermatitis 1996;35:234-6.

11. Fein, H. Stevens-Johnson syndrome. N Engl J Med 2005;352;1696.

Correspondence to: Dr. Catherine St-Pierre, Centre Hospitalier Universitaire de Sherbrooke, 3001, 12e Avenue Nord, Sherbrooke QC J1H 5N4; fax 819 820-6406; Catherine.St-Pierre@USherbrooke.ca 